\title{
On indices of meromorphic 1-forms
}

\author{
W. Ebeling and S. M. Gusein-Zade
}

\begin{abstract}
We discuss meromorphic 1-forms on compact complex manifolds and on complete intersections with isolated singularities. We give a Poincaré-Hopf type formula for them, i.e. we express the Euler characteristic in terms of singularities of a meromorphic 1-form. For that we introduce a suitable notion of an index of a germ of a meromorphic 1-form (with an additional structure) on an isolated complete intersection singularity. For a meromorphic 1-form on a complete intersection $V$ with isolated singularities the indices of the singular points sum up to (plus-minus) the Euler characteristic of a smoothing of $V$.
\end{abstract}

\section{Introduction}

For an isolated zero of a germ of a holomorphic vector field on a (smooth) complex manifold the notion of its index (or multiplicity) is defined. For a germ of a vector field $X=\sum_{i=1}^{n} X_{i}(x) \partial / \partial x_{i}$ on $\left(\mathbb{C}^{n}, 0\right)$ it is equal to the dimension of the local algebra $\mathcal{O}_{\mathbb{C}^{n}, 0} /\left(X_{1}, \ldots, X_{n}\right)$. If one has a holomorphic vector field with isolated zeros on a compact complex manifold $M^{n}$, then the sum of the indices of all zeros is equal to the Euler characteristic $\chi(M)$ of the manifold, i.e. to the characteristic number $c_{n}(T M)[M]$ (TM is the tangent bundle of $\left.M\right)$. Since non-zero holomorphic vector fields (as well as holomorphic 1-forms) on a compact complex manifold rarely exist, it is interesting to consider meromorphic vector fields of which there are a lot (at least for projective manifolds). For a meromorphic vector field defined by a holomorphic section of the vector bundle $T M \otimes L(L$ is a holomorphic line bundle on $M$ ), the sum of the indices of its zeros is equal to the corresponding characteristic number $c_{n}(T M \otimes L)[M]$ of the vector bundle $T M \otimes L$ (see e.g. [BB70]).

For a germ of a holomorphic 1-form $\alpha=\sum_{i=1}^{n} A_{i}(x) d x_{i}$ on $\left(\mathbb{C}^{n}, 0\right)$ its index $\operatorname{ind}_{0} \alpha$ is defined in the same way; it is equal to $\operatorname{dim} \mathcal{O}_{\mathbb{C}^{n}, 0} /\left(A_{1}, \ldots, A_{n}\right)$. For a holomorphic 1-form with isolated zeros on a compact manifold $M$, the sum of the indices of its zeros is equal to the characteristic number $c_{n}\left(T^{*} M\right)[M]=(-1)^{n} \chi(M)$, where $T^{*} M$ is the cotangent bundle (a Poincaré-Hopf type formula).

Let $\left(V^{n}, 0\right) \subset\left(\mathbb{C}^{n+k}, 0\right)$ be a germ of an isolated complete intersection singularity (ICIS) defined by equations $f_{1}=\cdots=f_{k}=0$, and let $\alpha$ be a holomorphic 1 -form on $(V, 0)$ (i.e., the restriction of a germ of a holomorphic 1-form $\sum_{i=1}^{n+k} A_{i}(x) d x_{i}$ on $\left.\left(\mathbb{C}^{n+k}, 0\right)\right)$ such that $\alpha$ has an isolated singular point at the origin on $V$. In this situation the index $\operatorname{ind}_{V, 0} \alpha$ of the 1-form $\alpha$ was defined in [EG01, EG02]. It was shown that it is equal to the dimension of the factor-algebra of the algebra $\mathcal{O}_{\mathbb{C}^{n+k}, 0}$ of germs of functions on $\left(\mathbb{C}^{n+k}, 0\right)$ factorized by the ideal generated by $f_{1}, \ldots, f_{k}$ and the $(k+1) \times(k+1)$ -

Received 14 March 2002, accepted in final form 24 July 2002.

2000 Mathematics Subject Classification 32Q55, 14B05, 32A20.

Keywords: meromorphic 1-forms, singular points, indices.

Partially supported by the DFG-programme 'Global methods in complex geometry', grants RFBR-01-01-00739, NWO-RFBR-047.008.005, INTAS-00-259, NSh-1272.2003.1.

This journal is (C) Foundation Compositio Mathematica 2004. 
minors of the matrix

$$
\left(\begin{array}{ccc}
\frac{\partial f_{1}}{\partial x_{1}} & \cdots & \frac{\partial f_{1}}{\partial x_{n+k}} \\
\vdots & \ddots & \vdots \\
\frac{\partial f_{k}}{\partial x_{1}} & \cdots & \frac{\partial f_{k}}{\partial x_{n+k}} \\
A_{1} & \cdots & A_{n+k}
\end{array}\right)
$$

Let $V^{n}$ be a complete intersection in $\mathbb{C P}^{n+k}$ with isolated singularities and let $\alpha$ be a holomorphic 1-form on $V$ (which means that the germ of $\alpha$ at each point is holomorphic). Singular points of $\alpha$ are singular points of the variety $V$ itself and zeros of $\alpha$ on the smooth part of $V$. Suppose that the singular points of the 1 -form $\alpha$ are isolated. Then the sum of their indices is equal to the characteristic number $c_{n}\left(T^{*} \widetilde{V}\right)[\widetilde{V}]=(-1)^{n} \chi(\widetilde{V})$ of a smoothing $\widetilde{V}$ of the variety $V$.

Let $\alpha$ be a meromorphic 1-form on a compact complex manifold $M^{n}$ which means that $\alpha$ is a holomorphic 1-form outside of a positive divisor $D$ and in a neighbourhood of each point of $M$ the form $\alpha$ can be written as $\widehat{\alpha} / F$ where $F=0$ is a local equation of the divisor $D$ and $\widehat{\alpha}$ is a holomorphic 1-form. Let $L$ be the line bundle associated to the divisor $D$, i.e. $L$ has a holomorphic section $s$ with zeros on $D$. Then $\omega=s \alpha$ is a holomorphic section of the vector bundle $T^{*} M \otimes L$. In some constructions (say, as in [BB70]) one defines a meromorphic 1-form on $M$ simply as a holomorphic section of the tensor product $T^{*} M \otimes L$ for a holomorphic line bundle $L$. This definition is somewhat different from the one formulated above. For example, in this case only the class of the divisor of poles of a meromorphic 1-form is defined, not the divisor itself. Moreover, in this setting, the value of a meromorphic 1-form on a vector field is not a function, but a section of the line bundle $L$. In the sequel we use the notation $\omega$ for a section of the vector bundle $T^{*} M \otimes L$, for short calling it a meromorphic 1-form as well. Suppose that the section $\omega$ has isolated zeros. Then the sum of their indices is equal to the characteristic number $c_{n}\left(T^{*} M \otimes L\right)[M]$ and thus depends on $L$.

For a meromorphic 1-form on a smooth compact complex curve $M$ the characteristic number $c_{1}\left(T^{*} M\right)[M]=-\chi(M)$ is equal to the number of zeros minus the number of poles counted with multiplicities. Therefore, to express the Euler characteristic of a compact manifold in terms of singularities of a meromorphic 1-form one has to take the divisor $D$ of its poles into account as well. If a meromorphic 1-form on a manifold $M^{n}$ is defined simply as a section of $T^{*} M \otimes L$, the divisor of poles is not defined and thus one also cannot define singular points of the form on its pole locus.

The aim of this paper is to give a Poincaré-Hopf type formula for meromorphic 1-forms, i.e. to express the Euler characteristic of a compact manifold or of a smoothing of a complete intersection in terms of singularities of a meromorphic 1-form. For that we introduce a suitable notion of an index of a germ of a meromorphic 1-form (with an additional structure) on an ICIS, so that the indices of the singular points sum up to (plus-minus) the Euler characteristic of a smoothing. There are several descriptions of the index of a meromorphic 1-form, one of them being the alternating sum of the dimensions of certain algebras.

\section{Singular points of meromorphic 1-forms}

A Poincaré-Hopf type formula can be considered as one describing a localization of an invariant (say, of the Euler characteristic) of a manifold at singular points of, say, a vector field or a 1-form, i.e. a representation of the invariant as the sum of integer invariants ('indices') corresponding to singular points. Therefore one first has to define singular points. Let $M^{n}$ be a complex manifold, let $L$ be a line bundle on $M$, and let $\omega$ be a holomorphic section of $T^{*} M \otimes L$. One has zeros of $\omega$ on $M$, but the pole locus of $\omega$ is not well defined. In order to discuss singular points of $\omega$ on its 


\section{ON INDICES OF MEROMORPHIC 1-FORMS}

pole locus (which is necessary as we saw in the example when $M$ was a curve), we have to fix this locus. This means that we have to choose a holomorphic section $s=s_{1}$ of the line bundle $L$ or to choose its zero divisor $D=D_{1}$. One can say that we have to consider $\alpha=\omega / s$, i.e. to proceed with our initial definition of a meromorphic 1-form.

For the further setting we suppose that the divisor $D$ of poles of the 1-form $\omega$ is non-singular (in particular, reduced). It is not very essential for this section but makes the discussion simpler. Since $D$ is a submanifold of $M$, there is a well defined map $\left.T^{*} M\right|_{D} \rightarrow T^{*} D$ and thus $\left.\left(T^{*} M \otimes L\right)\right|_{D} \rightarrow$ $\left.T^{*} D \otimes L\right|_{D}$ (the restriction of meromorphic 1-forms to $D$ ). (Using the 1-form $\alpha$, one can also define only a section of the vector bundle $\left.T^{*} D \otimes L\right|_{D}$, but not a meromorphic 1-form on $D$ with precisely defined pole locus.)

Let $\omega_{1}$ be the restriction of $\omega$ to $D_{1}$. It is a holomorphic section of the vector bundle $\left.T^{*} D \otimes L\right|_{D}$. Its zeros are well defined and should be considered as singular points of the meromorphic 1-form $\omega$ on the pole locus. To discuss its singular points on its pole locus we again have to fix a divisor. Suppose that there exists a (positive) divisor $D_{2}$ on $M$ which is the zero locus of another section $s_{2}$ of the same line bundle $L$ and which intersects $D_{1}$ transversally (in particular, this means that $D_{2}$ is non-singular at its intersection points with $D_{1}$ and $D_{1} \cap D_{2}$ is non-singular as well). One has the restriction of $\omega$ to $D_{1} \cap D_{2}$ and its zeros there.

Going on in this way we arrive at the situation when we have fixed $n$ divisors $D_{1}, \ldots, D_{n}$ (zeros of sections $s_{1}, \ldots, s_{n}$ of the line bundle $\left.L\right)$ so that, for each $i=1, \ldots, n, D_{1} \cap \cdots \cap D_{i}$ is non-singular. The set of singular points of the 1-form $\omega$ is the union of the zeros of $\omega$ itself and of the restrictions of $\omega$ to $D_{1} \cap \cdots \cap D_{i}$ for all $i=1, \ldots, n$. (For $i=n$ the intersection $D_{1} \cap \cdots \cap D_{n}$ is zero-dimensional and all its points should be considered as zeros.) One can say that we have to consider a collection of meromorphic 1-forms $\omega / s_{1}, \ldots, \omega / s_{n}$ on $M$ proportional to each other.

\section{A Poincaré-Hopf type formula for meromorphic 1-forms}

Let $M^{n}$ be a compact complex manifold, let $\omega$ be a meromorphic 1-form on $M$, that is, a holomorphic section of the bundle $T^{*} M \otimes L$ where $L$ is a holomorphic line bundle with non-zero holomorphic sections. Suppose that $D_{1}=D, D_{2}, \ldots, D_{n}$ are zero divisors of holomorphic sections of the line bundle $L$ such that, for each $i=1, \ldots, n, D_{1} \cap \cdots \cap D_{i}$ is non-singular. Suppose that the form $\omega$ itself and its restrictions to the submanifolds $D_{1} \cap \cdots \cap D_{i}, i=1, \ldots, n$, have only isolated zeros. Let $m_{0}$ (respectively $m_{i}, i=1, \ldots, n$ ) be the number of zeros of the form $\omega$ (respectively, of the restriction of $\omega$ to the intersection $D_{1} \cap \cdots \cap D_{i}$ ) counted with multiplicities. In particular $m_{n}$ is the number of points in $D_{1} \cap \cdots \cap D_{n}$.

Theorem 1. We have

$$
c_{n}\left(T^{*} M\right)[M]=(-1)^{n} \chi\left(M^{n}\right)=m_{0}-m_{1}+\cdots+(-1)^{n} m_{n} .
$$

Proof. The proof will use induction on the dimension $n$. We suppose that

$$
c_{n-1}\left(T^{*} D\right)[D]=(-1)^{n-1} \chi(D)=m_{1}-m_{2}+\cdots+(-1)^{n-1} m_{n} .
$$

One has $m_{0}=c_{n}\left(T^{*} M \otimes L\right)[M]$. For the characteristic class $c_{n}\left(T^{*} M \otimes L\right)$ one has

$$
c_{n}\left(T^{*} M \otimes L\right)=c_{n}\left(T^{*} M\right)+c_{1}(L) c_{n-1}\left(T^{*} M\right)+\cdots+c_{1}(L)^{n} .
$$

However, the normal bundle to $D=D_{1}$ in $M$ is isomorphic to the restriction $\left.L\right|_{D}$ and therefore $c\left(\left.T^{*} M\right|_{D}\right)=c\left(T^{*} D\right)\left(1-c_{1}\left(\left.L\right|_{D}\right)\right)$. Let $j: D \hookrightarrow M$ be the inclusion. Then

$$
\begin{aligned}
c\left(T^{*} D\right) & =j^{*}\left(c\left(T^{*} M\right)\left(1-c_{1}(L)\right)^{-1}\right), \\
c_{n-1}\left(T^{*} D\right) & =j^{*}\left(c_{n-1}\left(T^{*} M\right)+c_{1}(L) c_{n-2}\left(T^{*} M\right)+\cdots+c_{1}(L)^{n-1}\right) .
\end{aligned}
$$


Therefore

$$
\begin{aligned}
c_{n-1} & \left(T^{*} D\right)[D] \\
& =j^{*}\left(c_{n-1}\left(T^{*} M\right)+c_{1}(L) c_{n-2}\left(T^{*} M\right)+\cdots+c_{1}(L)^{n-1}\right)[D] \\
& =\left(c_{1}(L)\left(c_{n-1}\left(T^{*} M\right)+c_{1}(L) c_{n-2}\left(T^{*} M\right)+\cdots+c_{1}(L)^{n-1}\right)\right)[M] \\
& =\left(c_{n}\left(T^{*} M \otimes L\right)-c_{n}\left(T^{*} M\right)\right)[M] \\
& =m_{0}-c_{n}\left(T^{*} M\right)[M] .
\end{aligned}
$$

So

$$
c_{n}\left(T^{*} M\right)[M]=m_{0}-c_{n-1}\left(T^{*} D\right)[D]=m_{0}-m_{1}+\cdots+(-1)^{n} m_{n} .
$$

Remark 1. From the proof of Theorem 1 it follows that

$$
m_{0}=(-1)^{n}(\chi(M)-\chi(D))=(-1)^{n} \chi(M \backslash D) .
$$

Suppose that all zeros of $\omega$ are outside of $D$ (one can consider this situation as the generic one). Let $s$ be the holomorphic section of the line bundle $L$ with zeros on $D$. Then $\alpha=\omega / s$ is a holomorphic 1-form on $M \backslash D$ with simple poles along $D$. So in this case the number $m_{0}$ of zeros of the holomorphic 1-form $\alpha$ on $M \backslash D$ coincides with $(-1)^{n} \chi(M \backslash D)$. This is the relation which holds for holomorphic 1-forms on compact manifolds ( $M \backslash D$ is not compact).

Remark 2. In [BB70] there were considered meromorphic vector fields on complex manifolds. To have a version of Theorem 1 for vector fields it is necessary to define zeros of a vector field on its pole divisor. However (in contrast to a 1-form), a restriction of a vector field to a submanifold is not defined (as a vector field on the submanifold). One can use the projection of a vector field to the submanifold for that. However, this operation is not complex analytic and thus after projecting one has to consider zeros of non-meromorphic vector fields. These zeros should have signs and should be counted with them.

Example. Let

$$
\alpha=\frac{x d y-y d x+d z}{x^{2}+4 y^{2}+z^{2}+1}
$$

be a meromorphic 1 -form on the projective space $\mathbb{C P}^{3}(x, y, z$ are affine coordinates). One can see that the zeros of the corresponding $\omega$ on $\mathbb{C P}^{3}$ and also the zeros of $\left.\omega\right|_{D}, D=D_{1}=\left\{x^{2}+4 y^{2}+z^{2}+1=\right.$ $0\}$, are isolated and $m_{0}=0, m_{1}=4$. To define other singular points one has to choose $D_{2}$ and $D_{3}$ (e.g., $D_{2}=\left\{x^{2}+y^{2}+4 z^{2}=0\right\}, D_{3}=\left\{x^{2}+y^{2}+z^{2}=0\right\}$ ). One has $m_{2}=8, m_{3}=8$, $0-4+8-8=-4=(-1)^{3} \chi\left(\mathbb{C P}^{3}\right)$. Pay attention that as a meromorphic 1 -form on $\mathbb{C P}^{3}$ with poles on the hypersurface $P_{d}(x, y, z)=0, \operatorname{deg} P_{d}=d$, it is natural to take

$$
\frac{A_{d-2} d x+B_{d-2} d y+C_{d-2} d z}{P_{d}}
$$

where $A_{d-2}, B_{d-2}$, and $C_{d-2}$ are polynomials in $x, y, z$ of degree $d-2$ ( $\alpha$ is not of this form). However, one can show that such a form has non-isolated zeros on $\mathbb{C P}^{3}$ (at infinity).

\section{Meromorphic 1-forms on ICIS}

Let $V^{n}$ be a compact subvariety of a complex manifold $M^{n+k}$ such that in a neighbourhood of each point $V$ is a complete intersection in $M$ with only isolated singularities ( $M$ is not supposed to be compact). Let $\omega$ be a meromorphic 1-form on $M$, i.e. a holomorphic section of the bundle $T^{*} M \otimes L$, where $L$ is a line bundle on $M$ with non-zero holomorphic sections. By a smoothing $\widetilde{V}$ of $V$ we understand a smooth $\left(C^{\infty}\right)$ manifold which is obtained from $V$ by smoothing its singular points (which are ICIS) in the usual way (as complex ICIS). (If $V$ is a complete intersection in the projective space $\mathbb{C} P^{n+k}$, a smoothing of $V$ can be obtained as an analytic submanifold of $\mathbb{C P}^{n+k}$ as well.) 


\section{ON INDICES OF MEROMORPHIC 1-FORMS}

We want to formulate a Poincaré-Hopf type formula for the Euler characteristic of $V$ and of its smoothing $\widetilde{V}$ in terms of singular points of the 1-form $\omega$ on $V$. As above we suppose that there exist $n$ holomorphic sections $s_{1}, \ldots, s_{n}$ of the line bundle $L$ with zero divisors $D_{1}, \ldots, D_{n}$ such that, for each $i=1, \ldots, n$, the intersection $V \cap D_{1} \cap \cdots \cap D_{i}$ is of dimension $n-i$ and has only isolated singularities (and thus is reduced for $i<n$ ). We also suppose that the restriction of $\omega$ to the nonsingular part of $V$ and its restriction to the non-singular part of $V \cap D_{1} \cap \cdots \cap D_{i}(i=1, \ldots, n)$ have only isolated zeros. As singular points of the 1-form $\omega$ on $V$ we consider all zeros of its restrictions to the non-singular parts of $V$ and of the intersections $V \cap D_{1} \cap \cdots \cap D_{i}(i=1, \ldots, n)$ and all singular points of $V$ and of $V \cap D_{1} \cap \cdots \cap D_{i}$ as well.

Let $P$ be a singular point of the 1 -form $\omega$ on $V$. Suppose that the divisors $D_{1}, \ldots, D_{\ell}(0 \leqslant \ell \leqslant n)$ pass through the point $P$ and (if $\ell<n) D_{\ell+1}$ does not. In a neighbourhood of the point $P$ in some local coordinates on $M$ centred at the point $P$ we have the following situation. The variety (ICIS) $V$ (possibly non-singular) is defined by $k$ equations $f_{1}=\cdots=f_{k}=0$. Let divisors $D_{1}, \ldots, D_{\ell}$ be defined by equations $f_{k+1}=0, \ldots, f_{k+\ell}=0$. Let us recall that $\left\{f_{1}=\cdots=f_{k}=f_{k+1}=\cdots=\right.$ $\left.f_{k+i}=0\right\}$ is an ICIS for each $i=0,1, \ldots, \ell$. After choosing a local trivialization of the line bundle $L$, the 1 -form $\omega$ can be written as $\sum_{i=1}^{n+k} A_{i}(x) d x_{i}$ where $A_{i}(x)$ are holomorphic. One can say that we consider a collection of meromorphic 1 -forms $\omega / f_{k+1}, \ldots, \omega / f_{k+\ell}$ on $V$ proportional to each other. Let us denote the set of local data $\left(V, \omega, f_{k+1}, \ldots, f_{k+\ell}\right)$ by $\Omega$.

Let ind ${ }^{(0)} \Omega$ (respectively ind ${ }^{(i)} \Omega, i=1, \ldots, \ell$ ) be the index of the holomorphic 1-form $\omega$ on $V$ (respectively on the ICIS $V \cap D_{1} \cap \cdots \cap D_{i}$ ) defined in [EG01, EG02].

DeFinition. The alternating sum

$$
\operatorname{ind}_{P} \Omega=\operatorname{ind}^{(0)} \Omega-\operatorname{ind}^{(1)} \Omega+\cdots+(-1)^{\ell} \operatorname{ind}^{(\ell)} \Omega
$$

is called the index of the 1 -form $\omega$ at the point $P$ with respect to the divisors $D_{1}, \ldots, D_{n}$.

According to [EG01, EG02] one has the following three equivalent descriptions of the index $\operatorname{ind}_{P} \Omega$.

1) Let $B_{\delta}$ be the ball of sufficiently small radius $\delta$ centred at the origin in $\mathbb{C}^{n+k}$ and let $S_{\delta}$ be its boundary. Let $\varepsilon=\left(\varepsilon_{1}, \ldots, \varepsilon_{k}, \ldots, \varepsilon_{k+\ell}\right) \in\left(\mathbb{C}^{k+\ell}, 0\right)$ be small enough and such that $\varepsilon^{(k+i)}=\left(\varepsilon_{1}, \ldots, \varepsilon_{k}, \ldots, \varepsilon_{k+i}\right)$ is not a critical value of the map $F_{k+i}=\left(f_{1}, \ldots, f_{k}, \ldots, f_{k+i}\right)$ : $\left(\mathbb{C}^{n+k}, 0\right) \rightarrow\left(\mathbb{C}^{k+i}, 0\right)$ for each $i=0,1, \ldots, \ell$. The restriction of the 1 -form $\omega$ to the smooth manifold $V_{i}=F_{k+i}^{-1}\left(\varepsilon^{(k+i)}\right)$ has isolated zeros in $B_{\delta}$. Let $m_{i}$ be their number counted with multiplicities. Then ind $^{(i)} \Omega=m_{i}$, ind ${ }_{P} \Omega=m_{0}-m_{1}+\cdots+(-1)^{\ell} m_{\ell}$.

2) Let $K_{i}(i=0,1, \ldots, \ell)$ be the link of the ICIS $V \cap D_{1} \cap \cdots \cap D_{i}$, i.e. the intersection $V \cap D_{1} \cap$ $\cdots \cap D_{i} \cap S_{\delta}$. Let $d_{i}$ be the degree of the map

$$
\left(\omega, d f_{1}, \ldots, d f_{k}, \ldots, d f_{k+i}\right): K_{i} \rightarrow W_{k+i+1}\left(\mathbb{C}^{n+k}\right)
$$

$\left(W_{k+i+1}\left(\mathbb{C}^{n+k}\right)\right.$ is the Stiefel manifold of $(k+i+1)$-frames in the dual $\left.\mathbb{C}^{n+k}\right)$. Then ind ${ }^{(i)} \Omega=d_{i}$, $\operatorname{ind}_{P} \Omega=d_{0}-d_{1}+\cdots+(-1)^{\ell} d_{\ell}$.

3) Let $J_{i}(i=0,1, \ldots, \ell)$ be the ideal of the ring $\mathcal{O}_{\mathbb{C}^{n+k}, 0}$ of germs of holomorphic functions of $n+k$ variables at the origin generated by $f_{1}, \ldots, f_{k}, \ldots, f_{k+i}$ and the $(k+i+1) \times(k+i+1)$-minors of the matrix

$$
\left(\begin{array}{ccc}
\frac{\partial f_{1}}{\partial x_{1}} & \cdots & \frac{\partial f_{1}}{\partial x_{n+k}} \\
\vdots & \ddots & \vdots \\
\frac{\partial f_{k+i}}{\partial x_{1}} & \cdots & \frac{\partial f_{k+i}}{\partial x_{n+k}} \\
A_{1} & \cdots & A_{n+k}
\end{array}\right)
$$




\section{W. Ebeling and S. M. Gusein-Zade}

$$
\text { Let } \nu_{i}=\operatorname{dim}_{\mathbb{C}} \mathcal{O}_{\mathbb{C}^{n+k}, 0} / J_{i} \text {. Then ind }{ }^{(i)} \Omega=\nu_{i}, \operatorname{ind}_{P} \omega=\nu_{0}-\nu_{1}+\cdots+(-1)^{\ell} \nu_{\ell} \text {. }
$$

Remark 3. In [GSV91] and [SS96] there is defined the index of a holomorphic vector field on an ICIS. One advantage of using 1-forms instead of vector fields is that a description of type 3) does not exist for the index of a vector field.

Let us return to the global situation described at the beginning of the section.

TheOREM 2. We have

$$
\sum_{P} \operatorname{ind}_{P} \Omega=(-1)^{n} \chi(\widetilde{V})
$$

where $\widetilde{V}$ is a smoothing of the variety $V$.

Proof. If there exist an analytic smoothing $\widetilde{V}$ of $V$ inside $M^{n+k}$ and analytic smoothings of the divisors $D_{1}, \ldots, D_{n}$ (i.e. sections $\widetilde{s}_{1}, \ldots, \widetilde{s}_{n}$ of the line bundle $L$ close to $s_{1}, \ldots, s_{n}$ for which their zero divisors $\widetilde{D}_{1}, \ldots, \widetilde{D}_{n}$ are such that, for each $i=1, \ldots, n$, the intersection $\widetilde{V} \cap \widetilde{D}_{1} \cap \cdots \cap \widetilde{D}_{i}$ is smooth), then the statement follows directly from Theorem 1 since the index of a singular point counts the alternating sum of numbers of zeros on a smoothing vanishing at the point (see the description 1) of the index above).

If such smoothings do not exist, then the statement is derived from the following arguments. One can easily see that the statement of Theorem 1 and its proof hold in the situation when the manifold $M$ is almost complex (i.e. has a complex structure in the tangent bundle) and the manifolds $D_{1} \cap \cdots \cap D_{i}, i=1, \ldots, n$ ( $D_{i}$ is the zero locus of a section $s_{i}$ of the complex line bundle $L$ ), are almost complex submanifolds of $M$ (i.e. their tangent spaces are complex subspaces of the tangent spaces to the manifold $M$ ). The only difference is that in this case the multiplicity of a zero of a complex 1-form $\omega$ (a section of the complex bundle $T^{*} M \otimes L$ ) or of its restriction to the intersection $D_{1} \cap \cdots \cap D_{i}$ is in general not positive. However, if the manifold $M$, the intersections $D_{1} \cap \cdots \cap D_{i}$ and the 1-form $\omega$ are in fact complex analytic in neighbourhoods of all the singular points of $\omega$, then all the multiplicities are in fact positive.

One can construct almost complex smoothings $\widetilde{V}$ and $\widetilde{V} \cap \widetilde{D}_{1} \cap \cdots \cap \widetilde{D}_{i}$ of the varieties $V$ and $V \cap D_{1} \cap \cdots \cap D_{i}(i=1, \ldots, n)$ and a complex 1-form $\widetilde{\omega}$ approximating $\omega$ in the following way. Let $P$ be a singular point of $V$ or of an intersection $V \cap D_{1} \cap \cdots \cap D_{i}$. Suppose that $P \in V \cap D_{1} \cap \cdots \cap D_{\ell}$, $P \notin V \cap D_{1} \cap \cdots \cap D_{\ell+1}$ (here $\ell$ may be equal to zero if $P \notin D_{1}$ ). Choose a small ball $B$ of radius $r_{0}$ in a coordinate neighbourhood of the point $P(P=0)$ so that inside this ball $V$ is given by equations $f_{1}=\cdots=f_{k}=0, D_{i}$ is given by an equation $g_{i}=0(i=1, \ldots, \ell)$, and there are no other singular points of the 1-form $\omega$ inside the ball $B$. Let $B^{\prime}$ be the ball of radius $r_{0} / 2$ and let $\theta(r)$ be a smooth monotonous function such that $0 \leqslant \theta(r) \leqslant 1, \theta(r)=1$ in a neighbourhood of $r_{0} / 2$, and $\theta(r)=0$ in a neighbourhood of $r_{0}$. Let $\varepsilon_{0} \in \mathbb{C}^{k}, \varepsilon_{1}, \ldots, \varepsilon_{\ell} \in \mathbb{C}$ be small enough and such that the collection $\left\{f=\varepsilon_{0}\right\},\left\{f=\varepsilon_{0}\right\} \cap\left\{g_{1}=\varepsilon_{1}\right\} \cap \cdots \cap\left\{g_{i}=\varepsilon_{i}\right\}(i=1, \ldots, \ell)$ is a complex analytic smoothing of the varieties $V, V \cap D_{1} \cap \cdots \cap D_{i}(i=1, \ldots, \ell)$. Let $\widetilde{V}$ and $\widetilde{D}_{i}, i=1, \ldots, \ell$, be submanifolds of $M$ which coincide with $V$ and $D_{i}$ respectively outside of the ball $B$ and which are given by the equations $f(z)=\theta(r(z)) \varepsilon_{0}$ and $g_{i}(z)=\theta(r(z)) \varepsilon_{i}$ respectively, where $r(z)$ is the length of $z$ in the coordinates in $B$.

The tangent space $T_{z_{0}} \widetilde{V}$ to the manifold $\widetilde{V}$ at a point $z_{0} \in \widetilde{V} \cap \overline{B \backslash B^{\prime}}$ is close to the tangent space $T_{z_{0}} V_{z_{0}}$ to the complex submanifold $V_{z_{0}}=\left\{f(z)=\theta\left(r\left(z_{0}\right)\right) \varepsilon_{0}\right\}$ at the same point. If $z_{0} \in$ $\widetilde{V} \cap \widetilde{D}_{1} \cap \cdots \cap \widetilde{D}_{i}$, then also the tangent space $T_{z_{0}}\left(\widetilde{V} \cap \widetilde{D}_{1} \cap \cdots \cap \widetilde{D}_{i}\right)$ is close to the tangent space $T_{z_{0}}\left(V_{z_{0}} \cap D_{1, z_{0}} \cap \cdots \cap D_{i, z_{0}}\right)$ where $D_{j, z_{0}}$ is the complex analytic submanifold $\left\{g_{j}(z)=\theta\left(r\left(z_{0}\right)\right) \varepsilon_{j}\right\}$. The spaces $T_{z_{0}} V_{z_{0}}$ and $T_{z_{0}}\left(V_{z_{0}} \cap D_{1, z_{0}} \cap \cdots \cap D_{i, z_{0}}\right)$ are complex vector subspaces of the tangent space $T_{z_{0}} M$. To define a complex structure on the tangent space $T_{z_{0}}(\widetilde{V})$ one can construct a (real linear) map $H_{z_{0}}$ from the tangent space $T_{z_{0}} M$ to itself close to the identity (and thus non-degenerate) 


\section{ON INDICES OF MEROMORPHIC 1-FORMS}

which sends $T_{z_{0}} \widetilde{V}$ to $T_{z_{0}} V_{z_{0}}$ and (if $\left.z_{0} \in \widetilde{V} \cap \widetilde{D}_{1} \cap \cdots \cap \widetilde{D}_{i}\right)$ the tangent space $T_{z_{0}}\left(\widetilde{V} \cap \widetilde{D}_{1} \cap \cdots \cap \widetilde{D}_{i}\right)$ to $T_{z_{0}}\left(V_{z_{0}} \cap D_{1, z_{0}} \cap \cdots \cap D_{i, z_{0}}\right)$. Such a map (smoothly dependent on $z_{0}$ ) can easily be constructed using a hermitian scalar product on $T M$.

The map $H_{z_{0}}$ induces a complex structure on the tangent space $T_{z_{0}}(\widetilde{V})$ so that $T_{z_{0}}\left(\widetilde{V} \cap \widetilde{D}_{1} \cap\right.$ $\left.\cdots \cap \widetilde{D}_{i}\right)$ is a complex subspace of it and also a complex 1-form $\widetilde{\omega}$ on $T_{z_{0}}(\widetilde{V})$. Since $H_{z_{0}}$ is close to the identity, $\widetilde{\omega}$ has no singular points on $\widetilde{V} \cap \overline{B \backslash B^{\prime}}$. Therefore the 'almost complex version' of Theorem 1 applied to $\widetilde{V}, \widetilde{D}_{1}, \ldots, \widetilde{D}_{n}, \widetilde{\omega}$ yields the result.

In order to get the Euler characteristic of the variety $V$ itself one has to correct the formula of Theorem 2 taking the Milnor numbers of the singular points of $V$ into account. For $P \in V$, let $\mu_{P}$ denote the Milnor number of the ICIS $V$ at the point $P$ (see e.g. [Loo84]). Note that, if $V$ is non-singular at $P$, then $\mu_{P}=0$.

Theorem 3. We have

$$
(-1)^{n} \chi(V)=\sum_{P}\left(\operatorname{ind}_{P} \Omega-\mu_{P}\right)
$$

Remark 4. The Milnor number $\mu_{P}$ can also be written as an alternating sum of indices of holomorphic 1-forms on several ICIS. One can say that the term $\mu_{P}$ corresponds to the difference between two possible definitions of the index as in [SS98, Proposition 1.4].

\section{Multiple pole divisor}

In some cases it is natural to consider the situation when the pole locus of the meromorphic 1-form $\omega$ is a multiple of an irreducible divisor. Examples are the following:

1) A polynomial 1-form on $\mathbb{C}^{n}$ is a meromorphic 1 -form on $\mathbb{C P}^{n}$ the pole locus of which is a multiple of the infinite hyperplane $\mathbb{C P}_{\infty}^{n-1}$.

2) Let $f$ be a meromorphic function with the pole locus $k D$ where $D$ is an irreducible divisor (i.e. in a neighbourhood of each point $f$ can be written as $\tilde{f} / F^{k}$ where $\tilde{f}$ is holomorphic and $F=0$ is a local equation of $D)$. Then its differential $d f$ is a meromorphic 1 -form with the pole locus $(k+1) D$ (i.e. in a neighbourhood of each point $d f$ can be written as $\widetilde{\omega} / F^{k+1}$ where $\widetilde{\omega}$ is a holomorphic 1-form). A polynomial function of degree $k$ on $\mathbb{C}^{n}$ is a meromorphic function on $\mathbb{C P}^{n}$ with the pole locus $k \mathbb{C P}_{\infty}^{n-1}$.

Let us give a version of Theorem 1 for this case. The fact that the pole divisor of a 1 -form is multiple means that the corresponding line bundle is a power of another one. Let $\omega$ be a meromorphic 1-form on a compact complex manifold $M^{n}$, that is, a holomorphic section of the bundle $T^{*} M \otimes L$, where $L=\lambda^{k}, k>1$. Suppose that $D_{1}=D, D_{2}, \ldots, D_{n}$ are zero divisors of holomorphic sections of the line bundle $\lambda$ such that, for each $i=1, \ldots, n, D_{1} \cap \cdots \cap D_{i}$ is non-singular and the form $\omega$ itself and its restrictions to $D_{1} \cap \cdots \cap D_{i}$ have isolated zeros. Let $m_{0}$ (respectively $m_{i}, i=1, \ldots, n$ ) be the number of zeros of the 1 -form $\omega$ (respectively of its restriction to $D_{1} \cap \cdots \cap D_{i}$ ) counted with multiplicities.

Theorem 4. We have

$$
c_{n}\left(T^{*} M\right)[M]=(-1)^{n} \chi(M)=m_{0}-k m_{1}+\cdots+(-1)^{n} k m_{n} .
$$

Proof. For $n=1$, i.e. for curves, the statement is obvious. Suppose that the statement holds for manifolds of dimension $n-1$, in particular,

$$
c_{n-1}\left(T^{*} D\right)[D]=m_{1}-k m_{2}+\cdots+(-1)^{n-1} k m_{n} .
$$


Since $m_{0}=c_{n}\left(T^{*} M \otimes L\right)[M], m_{1}=c_{n-1}\left(T^{*} D \otimes L\right)[D]$, to prove the formula it is sufficient to show that the number

$$
c_{n}\left(T^{*} M\right)[M]+c_{n-1}\left(T^{*} D\right)[D]-c_{n}\left(T^{*} M \otimes L\right)[M]+(k-1) c_{n-1}\left(T^{*} D \otimes L\right)[D]
$$

is equal to zero. In order to prove this let us suppose that

$$
c\left(T^{*} M\right)=\prod_{i=1}^{n}\left(1+\xi_{i}\right) .
$$

Let $c_{1}:=c_{1}(\lambda)$ and let $j: D \hookrightarrow M$ be the inclusion. Then

$$
\begin{aligned}
c\left(T^{*} D\right) & =j^{*}\left(\frac{\prod\left(1+\xi_{i}\right)}{1-c_{1}}\right), \\
c\left(T^{*} M \otimes L\right) & =\prod\left(1+\xi_{i}+k c_{1}\right), \\
c\left(T^{*} D \otimes L\right) & =j^{*}\left(\frac{\prod\left(1+\xi_{i}+k c_{1}\right)}{1+(k-1) c_{1}}\right)
\end{aligned}
$$

(everywhere the product is taken for $i=1, \ldots, n$ ). Since $j^{*}(a)[D]=c_{1} a[M]$, the expression (1) is equal to the residue of

$$
\frac{1}{t^{n+1}}\left(\prod\left(1+\xi_{i} t\right)+c_{1} t \frac{\prod\left(1+\xi_{i} t\right)}{1-c_{1} t}-\prod\left(1+\left(\xi_{i}+k c_{1}\right) t\right)+(k-1) c_{1} t \frac{\prod\left(1+\left(\xi_{i}+k c_{1}\right) t\right)}{1+(k-1) c_{1} t}\right)
$$

at $t=0$. The function (2) has simple poles at $t_{1}=1 / c_{1}$ and $t_{2}=-1 /(k-1) c_{1}$. One easily calculates the residues of (2) at $t_{1}, t_{2}$ and $\infty$. The residue at $\infty$ turns out to be equal to zero and the residues at $t_{1}$ and $t_{2}$ cancel. By the residue theorem the residue at the origin is equal to zero.

Remark 5. The proof of Theorem 4 does not work for $k=1$ directly (the corresponding expression which differs from (2) by absence of the last summand has non-zero residues at $t=1 / c_{1}$ and $t=\infty$ which cancel). Moreover, the proof of Theorem 1 is more straightforward. This is the reason why we kept it.

Remark 6. In [HS98, Theorem 3.1] there is given a formula for the sum of the residues corresponding to the singular points of the foliation on the projective plane $\mathbb{C P}^{2}$ given by $d f=0$, where $f$ is a polynomial function of degree $k$ on $\mathbb{C}^{2}$ (which defines a meromorphic function on $\mathbb{C P}^{2}$ ). In our terms this is the formula for the number $m_{0}$ of zeros of the meromorphic 1-form $d f$, the pole locus of which is $k+1$ times the infinite line. Thus Theorem 4 can be considered as a generalization of [HS98, Theorem 3.1] to higher dimensions and to meromorphic 1-forms which are not, in general, differentials of functions.

\section{ACKNOWLEDGEMENT}

The authors would like to thank the referee for useful comments.

\section{REFERENCES}

BB70 P. F. Baum and R. Bott, On the zeros of meromorphic vector fields, in Essays on topology and related topics (Mémoires dédiés à Georges de Rham) (Springer, New York, 1970), 29-47.

EG01 W. Ebeling and S. M. Gusein-Zade, On the index of a holomorphic 1-form on an isolated complete intersection singularity, Russ. Math. Dokl. 380 (2001), 458-461.

EG02 W. Ebeling and S. M. Gusein-Zade, Indices of 1-forms on an isolated complete intersection singularity, Mosc. Math. J. 3 (2003), no. 2, 439-455. 


\section{ON INDICES OF MEROMORPHIC 1-FORMS}

GSV91 X. Gómez-Mont, J. Seade and A. Verjovsky, The index of a holomorphic flow with an isolated singularity, Math. Ann. 291 (1991), 737-751.

HS98 T. Honda and T. Suwa, Residue formulas for meromorphic functions on surfaces, Ann. Fac. Sci. Toulouse Math. (6) 7 (1998), 443-463.

Loo84 E. J. N. Looijenga, Isolated singular points on complete intersections, London Math. Soc. Lecture Note Series, vol. 77 (Cambridge University Press, Cambridge, 1984).

SS96 J. Seade and T. Suwa, A residue formula for the index of a holomorphic flow, Math. Ann. 304 (1996), 621-634.

SS98 J. Seade and T. Suwa, An adjunction formula for local complete intersections, Int. J. Math. 9 (1998), $759-768$.

W. Ebeling ebeling@math.uni-hannover.de

Universität Hannover, Institut für Mathematik, Postfach 6009, D-30060 Hannover, Germany

S. M. Gusein-Zade sabir@mccme.ru

Moscow State University, Faculty of Mechanics and Mathematics, Moscow, 119992, Russia 